\title{
CONOCIMIENTO DEL CÁNCER DE MAMA EN ESTUDIANTES DE ENFERMERÍA
}

\author{
KNOWLEDGE ABOUT BREAST CANCER \\ AMONG NURSING STUDENTS
}

\section{CONHECIMENTO DO CÂNCER DE MAMA EM ESTUDANTES DE ENFERMAGEM}

\author{
Meybol SantibáÑez Ramírez* \\ Andrea Símbala Delgado** \\ Nathalie Valenzuela NúÑEZ*** \\ Ismael Morales Ojeda**** \\ Ramón Gelabert Santané*****
}

\section{RESUMEN}

Objetivo: Este estudio evaluó el conocimiento acerca del cáncer de mama en estudiantes de Enfermería de universidades de la ciudad de Chillán. Material y método: Estudio descriptivo, transversal en estudiantes de Enfermería de tercer y cuarto año, correspondientes a tres universidades de Chillán, Chile. La muestra estuvo conformada por 187 estudiantes, a quienes se les aplicó una encuesta sobre conocimiento respecto del cáncer de mama. Resultados: La mayoría de los estudiantes presentó conocimiento para distinguir signos síntomas, tratamientos y diagnósticos sobre el cáncer de mama como futuros profesionales de la salud. Con respecto a los factores de riesgo y protectores relacionados al cáncer de mama, se observó una dificultad para reconocerlos. Respecto a la realización de la mamografía, el 80,7\% indicó que se debía realizar antes de los 30 años, sin embargo, de acuerdo a las guías clínicas, ésta se debe realizar después de los 50 años cuando no existen antecedentes familiares. Conclusión: Los estudiantes manifiestan adquisición de conocimientos respecto de la guía clínica, pero con dificultad para identificar factores de riesgo, protectores, e información específica respecto del tratamiento. Es necesario fortalecer la educación acerca de la prevención de cáncer de mama en los programas de estudio del área de la salud y promover la promoción de la salud.

Palabras clave: Cáncer de mama; Factores de riesgo; Estudiantes de Enfermería; Conocimiento.

\footnotetext{
*Licenciado en Enfermería, Carrera de Enfermería, Universidad Adventista de Chile, Chillán, Chile.

Email: mey.santibanez@gmail.com

** Licenciado en Enfermería, Carrera de Enfermería, Universidad Adventista de Chile, Chillán, Chile.

Email: andreasimbala@unach.cl

*** Licenciado en Enfermería, Carrera de Enfermería, Universidad Adventista de Chile, Chillán, Chile.

Email: nathalievalenzuela@unach.cl

**** Enfermero, Carrera de Enfermería, Facultad Ciencias de la Salud. Universidad Adventista de Chile, Chillán, Chile. Email: ismaelmorales@unach.cl. Autor de correspondencia.

***** Médico, Académico Universidad Adventista de Chile, Chillán, Chile. Email: dirinvestigacion@unach.cl
} 


\begin{abstract}
Objective: This study assessed the knowledge about breast cancer among Nursing students from universities in the city of Chillán (Chile). Materials and methods: Descriptive, cross-sectional study carried out with third and fourth-year Nursing students from three universities of Chillán. The sample consisted of 187 students, who answered a survey related to their knowledge about breast cancer. Results: Most of the students demonstrated sufficient knowledge to distinguish signs and symptoms, treatments, and diagnoses of breast cancer as prospective healthcare practitioners. Difficulties regarding recognition of risk and protective factors related to breast cancer were observed. In relation to mammography tests, $80.7 \%$ indicated that they are performed before the age of 30. According to clinical guidelines, however, they should be performed after the age of 50, when there is no family history related to the disease. Conclusion: Students report knowledge of clinical guidelines, though it is difficult for them to identify risk and protective factors as well as specific treatment information. It is thus necessary to improve the level of education related to the prevention of breast cancer in study programs and encourage health promotion.
\end{abstract}

Key words: Breast cancer; Risk factors; Nursing students; Knowledge.

\title{
RESUMO
}

Objetivo: Este estudo avaliou o conhecimento sobre o câncer de mama em estudantes de Enfermagem das universidades da cidade de Chillán. Métodos: Estudo descritivo, transversal em estudantes de Enfermagem do terceiro e quarto ano, correspondente a três universidades em Chillán, Chile. A amostra foi composta por 187 estudantes que receberam um questionário sobre o conhecimento sobre o câncer de mama. Resultados: A maioria dos estudantes apresentou conhecimentos para distinguir os sinais, sintomas, tratamentos e diagnósticos sobre câncer de mama como profissionais da saúde. No entanto, os estudantes mostraram dificuldade em reconhecer exatamente quais eram os fatores de risco e fatores de proteção relacionados ao câncer de mama. Em relação à mamografia, 80,7\% indicaram que o exame devia ser feito antes dos 30 anos, porem as guias clínicas indicam que deve ser feito após 50 anos, quando não há história familiar. Conclusão: Os alunos manifestam a aquisição de conhecimento em relação à diretriz clínica, mas com dificuldade em identificar fatores de risco, protetores e informaçóes específicas sobre o tratamento. É necessário fortalecer a educação sobre a prevenção do câncer de mama nos programas de estudo da área da saúde e promover a promoção da saúde.

Palavras chave: Câncer de mama; Fatores de risco; Estudantes de Enfermagem; Conhecimento.

Fecha recepción: 22/06/2018 Fecha aceptación: 22/03/2019

\section{INTRODUCCIÓN}

El cáncer es una de las principales causas no transmisibles de morbilidad y mortalidad, con un registro durante el año 2015 de 14 millones de nuevos casos de cáncer en el mundo, falleciendo ese año 8,8 millones de personas, previéndose en los futuros 20 ańos un alza de $70 \%$ en el diagnóstico de cáncer $^{(1)}$. El cáncer de mama es el segundo cáncer más común en el mundo, constituyendo $25 \%$ de todos los cánceres y el cáncer más frecuente entre las mujeres, con una incidencia estimada de
1,67 millones de casos de cáncer diagnosticados en $2012^{(2,3)}$ y la segunda causa de muerte en mujeres a nivel mundial ${ }^{(1)}$. Los estudios epidemiológicos y de observación clínica demuestran que existen múltiples factores de riesgo para el posible desarrollo de cáncer de mama ${ }^{(4)}$, así como una alta tasa de mortalidad, que se revierte cuando se detecta oportunamente ${ }^{(5,6)}$.

En el mundo, el cáncer de mama es la quinta causa de muerte por cáncer en general con 522.000 muertes en el año 2012(2). En Sudamérica se aprecia un aumento de la mortalidad por cáncer 
de mama, estimándose que anualmente fallecerán 24.681 mujeres $^{(7)}$, describiéndose como problemas frecuentes la detección de cáncer en una fase avanzada como la falta de diagnóstico y tratamiento ${ }^{(5)}$. En el caso de Brasil, la incidencia de cáncer de mama es de 52 casos por 100.000 mujeres $^{(5,8)}$, en Colombia 35,7 casos por 100.000 habitantes $^{(6)}$. Chile presenta una tasa de mortalidad observada de 15,69 por 100.000 mujeres en $2012^{(7)}$; en el quinquenio 2009 a 2013 se observa que la mortalidad incrementa con la edad, colocándose esta enfermedad como la primera causa de muerte por cáncer en mujeres ${ }^{(9)}$, para finalmente en 2015 aumentar a 16,6 por cada 100.000 mujeres $^{(10)}$. Las regiones de Chile con mayor tasa ajustada de mortalidad por cáncer de mama son Magallanes con 16,9, Valparaíso con 14,3 y la Región Metropolitana con 14,00, muy por encima de la Región del Biobío con 12,7 $7^{(10)}$.

En Chile, la incidencia de cáncer se analiza a partir de los datos procedentes de hospitales públicos, pudiendo haber otros casos no registrados adecuadamente en fuerzas armadas e incluso en servicios delegados pertenecientes al sistema público de salud, lo que dificulta el registro de cáncer real de toda la población ${ }^{(11)}$. En el sector público se ha observado un aumento de la incidencia de los estadios 0, detectados por las mamografías, y los estadios avanzados como el III y IV se han mantenido estables. En cambio, en el sector privado se han incrementado los estadios 0 en un $20 \%$, y los estadios III y IV han disminuido en un 10 y $11 \%{ }^{(9)}$.

Frente a este contexto y a lo expresado en la Ley 19.966 del año 2004 que establece un Régimen de Garantías Explícitas en Salud ${ }^{(12)}$, el Ministerio de Salud redactó la Guía Clínica Chilena de Cáncer de Mama, guía que tiene como propósito mejorar la calidad de vida, y disminuir el costo monetario y emocional de las pacientes, bajo el estandarte de equidad en salud, incluyendo orientaciones para todo el equipo multidisciplinario relacionado con la atención de personas con cáncer de mama, incluyendo a estudiantes de pre y posgrado ${ }^{(7)}$.

Es en este propósito en donde está inserto el profesional de enfermería, quien cumple su rol dentro del equipo multidisciplinario, centrándose en aspectos relacionados con educación, prevención, promoción y cuidados de la salud, en los tres niveles de atención donde ejecutan la gestión del cuidado ${ }^{(5)}$. El cuidado consiste en tomar contacto con el otro, elemento esencial en la relación enfermero-paciente, donde se busca empatizar o sentir desde el paciente; lo que representa la principal característica de la relación profesional ${ }^{(13)}$.

Frente a esta realidad, se precisa que los estudiantes de Enfermería conozcan acerca de los aspectos relacionados con el manejo del cáncer de mama. Tal es la importancia de esto, que la Comisión Nacional de Acreditación espera que los estudiantes desarrollen, desde su perfil profesional, la capacidad de proporcionar una atención integral al individuo, familia y comunidad, tanto que estén sanas o enfermas y en las diferentes etapas del ciclo vital; una atención basada en la promoción, prevención, recuperación, rehabilitación de la salud y en la etapa terminal de la vida ${ }^{(14)}$.

Debido a lo anterior se plantea como objetivo de este estudio evaluar el conocimiento acerca del cáncer de mama presente en estudiantes de Enfermería de universidades de la ciudad de Chillán.

\section{MATERIAL Y MÉTODO}

Estudio descriptivo, transversal, realizado en estudiantes de las carreras de Enfermería de tres universidades de la ciudad de Chillán, Chile, durante el año 2017.

La muestra fue de tipo no probabilística según disponibilidad e incluyó a 187 estudiantes de tercer y cuarto año de las carreras de Enfermería, considerándose que éstos ya tienen una experiencia clínica antes del ingreso a las prácticas finales de la carrera. Los criterios de inclusión fueron: estudiantes matriculados en la carrera correspondiente a $3^{\text {ro }}$ y $4^{\text {to }}$ año, que hayan realizado prácticas clínicas en distintas asignaturas, excluyéndose aquellos estudiantes que no completaron el consentimiento o el cuestionario en su totalidad, estaban en pasantías, o tenían inasistencia o licencia médica el día de la aplicación de la encuesta.

La encuesta autoadmistrada se centró en la labor de enfermería, particularmente en la promoción y prevención de la salud. En la encuesta se debía responder preguntas sobre conocimiento del cáncer de mama: factores protectores, factores de riesgo, signos y síntomas, tamizajes y práctica del autoexamen de mamas. La encuesta se creó a partir de los contenidos de la Guía Clínica Cáncer de Mama, 2015 ${ }^{(7)}$. Guía elaborada por el Ministerio 
de Salud de Chile en el año 2004 y actualizada en 2010 y 2015, en la cual se describe: la epidemiología del cáncer de mama, los pacientes y escenarios clínicos donde se entrega la atención de salud y en la cual se establecen las recomendaciones para la prevención, tamizaje, sospecha diagnóstica con estadificación, tratamiento y seguimiento durante y después de la enfermedad.

Las preguntas que hacen alusión a la experiencia, creencias respecto de cuáles son signos o síntomas, factores de riesgo, prevención, e intervención relacionadas con el cáncer de mama, incluidas aquellas preguntas sobre técnicas diagnósticas y tratamientos son de tipo dicotómicas con alternativas si/no, en las cuales el estudiante podía elegir su preferencia según el nivel de conocimiento que tuviese. Asimismo, para complementar la elaboración de la encuesta que se aplicó en esta investigación, se revisó la estructura de encuestas en las que se preguntaba por el conocimiento sobre cáncer de mama, realizada en dos estudios, uno en Argentina en el 2013 con mujeres entre 35 y 65 años y otro que se aplicó en Ecuador a estudiantes de primer año de enfermería en el 2014 .

Se realizó una validación por expertos, la que incluyó la consulta a cinco profesionales de la salud en el área de medicina, enfermería y obstetricia, todos académicos con experiencia en cuidados de la mujer o en oncología. A éstos se les suministró información respecto de los antecedentes del estudio, objetivos y contexto en que se aplicaría la encuesta. Se les entregó un cuadernillo que contenía cada pregunta por separado, en el cual evaluaron: 1) adecuación de las preguntas a las características de la muestra y 2) pertinencia de las preguntas respecto de los objetivos planteados, existiendo la posibilidad de sugerir cambios a cada pregunta por separado. Este proceso permitió identificar cuáles preguntas requerían modificaciones o clarificaciones. Además, para comprobar la claridad de las preguntas y su comprensión se realizó una prueba piloto con 30 estudiantes de Enfermería de una de las universidades.

El estudio fue aprobado por el Comité Ético Científico de la Universidad Adventista de Chile. La muestra se recolectó con autorización de las distintas entidades académicas en las universidades de Chillán. Previo a la aplicación de la encuesta, los participantes dieron por escrito su consentimiento informado.

El análisis estadístico se realizó con SPSS. v19. Se utilizó estadística descriptiva, donde las variables cuantitativas se expresaron mediante promedios y las variables cualitativas mediante tablas de frecuencia y porcentaje. El conocimiento se evaluó a partir de las respuestas correctas dadas por los estudiantes que fueron acordes a los criterios de la Guía Clínica de Cáncer de Mama. Las respuestas de cada estudiante fueron puntuadas en una escala de 1 a 100. Se determinó la normalidad de la variable conocimiento con la prueba de Kolmogorov-Smirnov y se utilizó la prueba t para determinar diferencias de puntaje según sexo. Se consideró como significancia estadística valor $p<0,05$.

\section{RESULTADOS}

El promedio de edad de los 187 estudiantes fue de 23 años, de sexo femenino un 75,4\%. El estado civil más frecuente es soltero con un $92 \%$. Un 9,1\% de los estudiantes tiene hijos.

Del total de estudiantes de Enfermería, un $97,3 \%$ no ha tenido la oportunidad de atender a pacientes con cáncer de mama, destacándose que conocen a estos pacientes según experiencias de la vida personal o familiar en un 39,6\% (Tabla 1). Los estudiantes en un $13,4 \%$ cree falsamente que entre más joven una mujer mayor riesgo de padecer cáncer de mama y 55,1\% responde que el sobrepeso antes de la menopausia no corresponde a un factor de alto riesgo (Tabla 2).

Los estudiantes de Enfermería indicaron que la frecuencia de realización del autoexamen de mama, según la Guía Clínica, es mensual en un 49,7\%, las respuestas erróneas, del 50,3\% restante, se distribuyeron en: $27,3 \%$ semanal, $16 \%$ anual, $4,8 \%$ semestral y $2,1 \%$ trimestral.

$\mathrm{Al}$ evaluar de manera dicotómica (si/no) cuáles son las creencias de los estudiantes respecto de técnicas diagnósticas, tratamiento y cuidados postoperatorios, las respuestas obtenidas fueron las siguientes: Los estudiantes, en un $71,1 \%$ indican la biopsia percutánea como la correcta técnica diagnóstica para el cáncer de mama. En un $96,8 \%$ la mamografía digital, Pet Scan en un $35,3 \%$, ecografía en un $67,4 \%$. Los estudiantes indican que el tratamiento para el cáncer de mama 
es la biopsia en un $15,0 \%$, mamografía en un $15 \%$, terapia hormonal en un 60,4\%, mastectomía parcial/total en un $97,3 \%$ y quimioterapia en un $98,4 \%$. Según las respuestas entregadas por los estudiantes, los cuidados que se deben tener con un paciente postoperado de cáncer de mama son: puncionar siempre el brazo del lado postoperado en un $11,8 \%$, no puncionar el brazo del lado mastectomizado en un 93,6\%, no tomar la presión arterial del brazo mastectomizado en un $85,6 \%$, y tomar gases arteriales del brazo del lado mastectomizado en un $15 \%$.

Con respecto al significado de la mastectomía, indican que es la extirpación de la mama en un $93,6 \%$, extirpación de un tumor benigno en un 4,3\%, y disección de ganglios linfáticos axilares en un $2,1 \%$. Al mismo tiempo, según las alternativas dadas en la encuesta, pudieron reconocer lo que es un linfedema en un $74,9 \%$

Al evaluar el puntaje de preguntas acertadas en una escala de 1 a 100 por sexo, los hombres obtuvieron $\overline{\mathrm{X}}=74,55 \quad \mathrm{DS}=6,5$ puntos, $\mathrm{y}$ el femenino $\bar{X}=75,26$ DS $=7,40$. La prueba $t$ no indicó diferencia significativa entre hombres y mujeres respecto del conocimiento del cáncer de mama $(p=0,563)$ (Gráfico 1).

Tabla 1. Experiencias, factores de riesgo y protectores, signos y síntomas de cáncer de mama, según los estudiantes de Enfermería en tres universidades de Chillán ( $\mathrm{n}=187)$.

$\mathrm{fx}^{\mathrm{Sí}} \% \quad \mathrm{fx} \quad \mathrm{No}$

Experiencia en relación al cáncer de mama

¿Durante tus prácticas de carrera tuviste la experiencia de otorgar cuidados de enfermería a personas con cáncer de mama?

En sus relaciones familiares o de amistad, ¿̇alguien allegado a usted tuvo cáncer de mama?

Las estudiantes indican que ellas realizan su autoexamen de mamas de manera periódica** $\left.^{* *} \mathrm{n}=141\right)$

55,3

$5 \quad 2,7 \quad 182 \quad 97,3$

$74 \quad 39,6 \quad 113 \quad 60,4$

Creencias acerca de cuáles son factores de riesgo del cáncer de mama

Menarquia precoz

$90 \quad 48,1^{*} \quad 97 \quad 51,9$

Alto consumo de carbohidratos

$\begin{array}{llll}60 & 32,1^{*} & 127 & 67,9\end{array}$

Factores medioambientales

$156 \quad 83,4^{*} \quad 31 \quad 16,6$

Tener familiares de 1er grado con cáncer de mama

$175 \quad 93,6^{*} \quad 12 \quad 6,4$

Uso de anticonceptivos

Antecedente de cáncer glandular

$102 \quad 54,5^{*} \quad 85 \quad 45,5$

$17493,0^{*} \quad 13 \quad 7$

Creencia acerca de cuáles son factores protectores del cáncer de mama

Realizarse una mamografía a los 30 años de edad sin antecedentes familiares de cáncer de mama

Realizarse un PAP a los 20 años de edad

Realizarse una mamografía antes de los 50 años de edad

Ser nulípara después de los 35 años de edad

Lactancia materna

$\begin{array}{rlrc}151 & 80,7 & 36 & 19,3^{*} \\ 90 & 48,1 & 97 & 51,9^{*} \\ 155 & 82,9^{*} & 32 & 17,1 \\ 30 & 16 & 157 & 84,0^{*} \\ 164 & 87,7^{*} & 23 & 12,3\end{array}$

Creencias acerca de cuáles son signos o síntomas característicos del cáncer de mama

Abultamiento mamario

\begin{tabular}{rcrl}
179 & $95,7^{*}$ & 8 & 4,3 \\
145 & $77,5^{*}$ & 42 & 22,5 \\
114 & $61,0^{*}$ & 73 & 39 \\
157 & $84,0^{*}$ & 30 & 16 \\
32 & 17,1 & 155 & $82,9^{*}$ \\
51 & 27,3 & 136 & $72,7^{*}$ \\
181 & $96,8^{*}$ & 6 & 3,2 \\
176 & $94,1^{*}$ & 11 & 5,9 \\
\hline
\end{tabular}

Asimetría de los pezones

Edema en brazo de mama afectada

Enrojecimiento mamario

Adormecimiento de las extremidades

Parestesias

Hundimiento del pezón o dolor en la zona

Secreción purulenta del pezón sin lactancia

*Respuestas correctas.

**Item estaba reservado para ser respondido por estudiantes de sexo femenino. 
Tabla 2. Intervenciones, profesional competente, personas de alto riesgo de cáncer de mama, según los estudiantes de Enfermería en tres universidades de Chillán ( $\mathrm{n}=187)$.

\begin{tabular}{rcrr}
\multicolumn{2}{c}{ Sí } & \multicolumn{2}{c}{ No } \\
fx & $\%$ & fx & $\%$ \\
& & & \\
187 & $100,0^{*}$ & 0 & 0 \\
76 & 40,6 & 111 & $59,4^{*}$ \\
147 & $78,6^{*}$ & 40 & 21,4 \\
184 & $98,4^{*}$ & 3 & 1,6 \\
182 & $97,3^{*}$ & 5 & 2,7 \\
183 & $97,9^{*}$ & 4 & 2,1
\end{tabular}

Intervenciones de enfermería en la prevención del cáncer de mama

Educar a la población

Solicitar exámenes de sangre

Derivar a especialista

Examen físico

Consultar por la realización de mamografía

Evaluar signos y síntomas

Creencia acerca de cuál es el profesional competente para diagnosticar cáncer de mama

Matrón/a

$96 \quad 51,39148,7^{*}$

Enfermero/a

$34 \quad 18,2 \quad 15381,8^{*}$

Ginecólogo/a

Médico especialista

$146 \quad 78,1 \quad 41 \quad 21,9^{*}$

Médico general

$179 \quad 95,7^{*} \quad 8 \quad 4,3$

$8646 \quad 10154,0^{*}$

Personas con alto riesgo de cáncer de mama

Radiografía de tórax antes de los 30 años

Familiar consanguíneo de cáncer en 1er o $2^{\circ}$ grado

$\begin{array}{lllll}72 & 38,5 & 115 & 61,5^{*}\end{array}$

Densidad mamográfica aumentada

$\begin{array}{llll}182 & 97,3^{*} & 5 & 2,7\end{array}$

A menor edad mayor riesgo

$16990,4^{*} \quad 18 \quad 9,6$

Sobrepeso antes de la menopausia

Obesidad centrípeta antes de la menopausia

$25 \quad 13,4 \quad 16286,6^{*}$

$84 \quad 44,9^{*} \quad 103 \quad 55,1$

$96 \quad 51,3^{*} \quad 91 \quad 48,7$

*Respuestas correctas.

Gráfico 1. Evaluación de conocimiento del cáncer de mama en estudiantes de Enfermería según sexo.

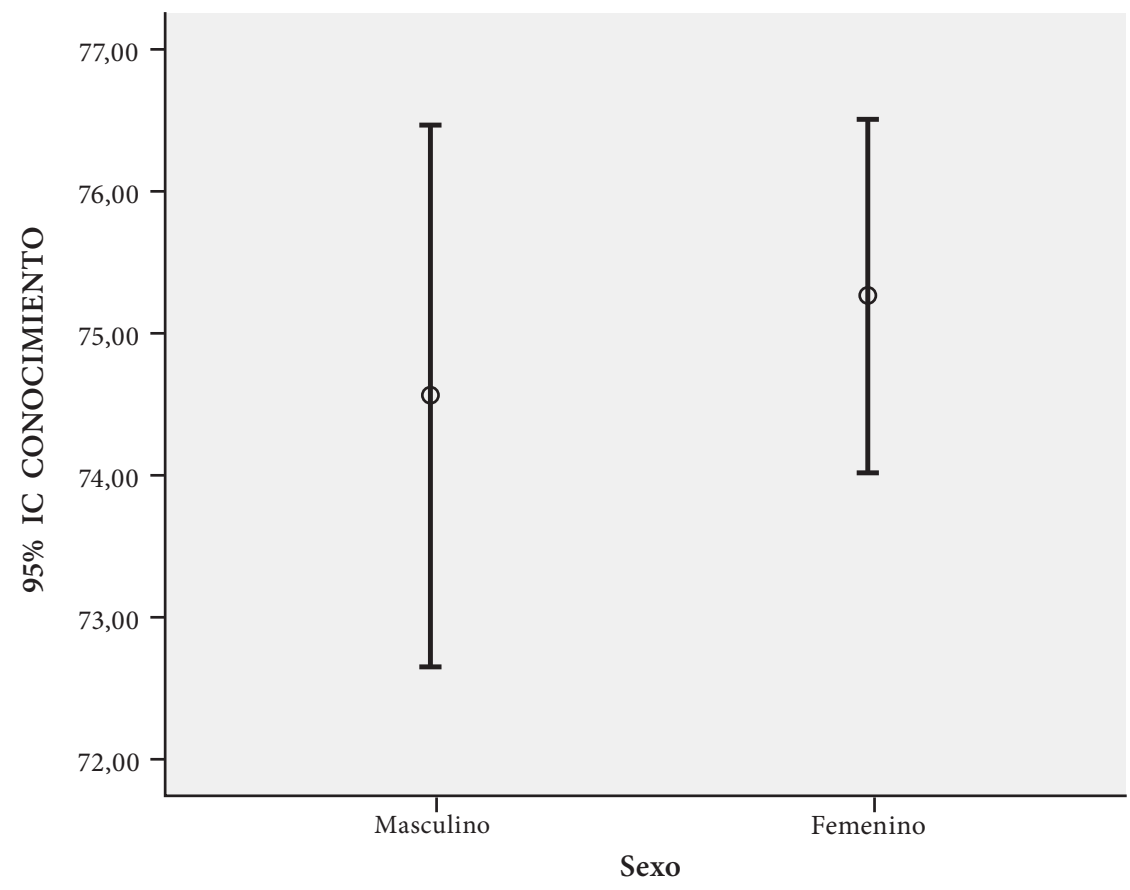




\section{DISCUSIÓN}

A pesar de ya haber realizado prácticas en algunas asignaturas y tener fundamentos teóricos acerca del cáncer de mama, los estudiantes de las distintas universidades no han tenido la oportunidad de atender a pacientes con cáncer de mama, y su enfrentamiento con este tipo de enfermedad se ha debido a experiencias de su vida personal o familiar. Debido a la elevada incidencia de este cáncer en nuestro país ${ }^{(9)}$, debería considerarse la posibilidad de que las universidades realicen una pasantía por un servicio especializado en oncología, con el fin de que se refuercen estos contenidos en virtud del diagnóstico temprano y el oportuno tratamiento.

Según Páez et al., existe dificultad para enseñar los factores de riesgo y protectores del cáncer de mama en una universidad de Colombia ${ }^{(15)}$. Aspecto importante a destacar en los planes de estudios, pues los estudiantes de Enfermería son también responsables de educar a la población en salud ${ }^{(5)}$. La falta de conocimiento sobre las edades para poder realizar la pesquisa con mamografía, es preocupante, ya que por sobre la mitad de los estudiantes no reconoce con qué frecuencia se debe realizar el autoexamen de mama, creyendo erróneamente que la frecuencia es semanal, o anual. Los estudiantes creen falsamente que entre más joven una mujer mayor riesgo de padecer cáncer de mama y que la alteración en el peso no tiene mayor relevancia en el desarrollo del cáncer de mama. Lo cual es contrario a lo que dice la evidencia científica que afirma que entre los principales factores de riesgo del cáncer se encuentra el sobrepeso, obesidad ${ }^{(16)}$ y la edad ${ }^{(7,17)}$.

Se aprecia dificultad para reconocer que toda sospecha de cáncer de mama se debe derivar a especialista, concordando con el estudio de Canicali et $a l^{\left({ }^{(8)}\right.}$; un número pequeño de estudiantes no identifica al médico especialista como el profesional competente para diagnosticar cáncer de mama, al igual que en los resultados de Prolla et al. ${ }^{(5)}$, lo cual es acorde con la dificultad de hacer diagnóstico temprano en países latinoamericanos ${ }^{(18)}$. Los estudiantes creen erróneamente que la mamografía y la ecografía respectivamente son técnicas diagnósticas en vez de técnicas de tamizaje.

En lo que se refiere al tratamiento, los estudiantes identificaron adecuadamente a la terapia hormonal, mastectomía y quimioterapia como tratamientos del cáncer de mama, concordando con Álvarez et al. ${ }^{(19)}$.
Los estudiantes tienen conocimiento sobre los cuidados postoperatorios en las mujeres con cáncer de mama, e incluso dos tercios de ellos identifica adecuadamente entre las alternativas de la encuesta lo que es un linfedema. La incorporación de este conocimiento frente a la patología es importante, principalmente frente a procedimientos de enfermería como el control de signos vitales o punciones, todos procedimientos que deben ser realizados en el brazo contrario al del lado mastectomizado ${ }^{(20)}$.

Finalmente, dentro del marco de formación profesional de la carrera de Enfermería, la importancia de la evaluación de conocimiento realizada está vinculada con la formación de un estudiante acorde a las necesidades del sistema de salud, que tiene capacidad de coordinarse con distintos profesionales en el contexto de mejorar la salud pública del país. Un estudiante que tempranamente adquiere desde el conocimiento las habilidades para prevenir, detectar, aconsejar e intervenir en enfermedades catastróficas o terminales, constituirá un profesional de enfermería empoderado, capaz de cumplir su objetivo, el cual es cuidar la salud y la vida con la máxima calidad posible, evitando complicaciones derivadas de la enfermedad, con capacidad de entregar apoyo, y contención biopsicosocioemocional a la persona enferma y su familia ${ }^{(21)}$

\section{CONCLUSIÓN}

El estudio reveló la necesidad de resaltar en los distintos planes de estudio de enfermería los contenidos asociados al cáncer de mama, siendo necesario programar en las prácticas espacios donde los estudiantes puedan hacer diagnóstico de enfermería e intervención respecto a la promoción de salud, prevención y tratamiento de la patología, puesto que el desconocimiento dificulta la oportuna derivación a especialistas y constituye un factor de riesgo para los usuarios del sistema de salud ${ }^{(18)}$.

Estas debilidades deben ser consideradas a partir de lo recomendado por la Guía Clínica del Cáncer de Mama que indica que también está orientada a los estudiantes de pregrado en Ciencias de la Salud ${ }^{(7)}$. A la hora de evaluar el conocimiento global respecto del cáncer de mama, se aprecia, en primer lugar, que los promedios obtenidos entre el grupo de hombres y mujeres son similares en ambos grupos, lo que indica que no hay 
diferencias significativas en cuanto a la adquisición de los contenidos de una patología prevalente en mujeres. En segundo lugar, respecto a experiencia de evaluación realizada se destaca la importancia de algunos conocimientos por sobre otros, los cuales no pueden ser ni omitidos en la enseñanza, ni desconocidos por los estudiantes, pues son indispensables respecto a la adquisición del rol de enfermería; conocimientos relacionados con la identificación de factores de riesgo o protección, diagnóstico temprano, y tratamiento, todos partes de la educación en salud que el profesional brinda a la sociedad.

La limitación del estudio fue la falta de estudios publicados sobre el tema de la enseñanza y adquisición de conocimiento respecto del cáncer de mama en estudiantes universitarios en Chile. Esto evidencia la necesidad de realizar investigaciones respecto de cómo las carreras de Enfermería imparten conocimiento a partir de las Guías Clínicas, y cómo éste es adquirido por los estudiantes, en un contexto de mejorar la salud pública del país.

\section{REFERENCIAS}

1. Ghoncheh M, Pournamdar Z, Salehiniya $\mathrm{H}$. Incidence and Mortality and Epidemiology of Breast Cancer in the World. Asian Pac J Cancer Prev [Internet]. 2016 [cited 2019 May 4]; 17(S3):43-46. Available from: https://www.ncbi. nlm.nih.gov/pubmed/27165206

2. Ferlay J, Soerjomataram I, Dikshit R, Eser S, Mathers C, Rebelo $\mathrm{M}$ et al. Cancer incidence and mortality worldwide: sources, methods and major patterns in GLOBOCAN 2012. Int J Cancer [Internet]. 2015 Mar 1 [cited 2019 May 4]; 136(5): E359-386. Available from: https://www.ncbi.nlm. nih.gov/pubmed/25220842

3. Moreno-González MM, Salazar-Maya AM, Tejada-Tayabas LM. Experiencia de cuidadores familiares de mujeres con cáncer de mama: una revisión integradora. Aquichan [Internet]. 2018 Mar [citado 4 May 2019]; 18(1): 56-68. Disponible en: http://dx.doi.org/10.5294/aqui.2018.18.1.6.

4. Sung H, Ren J, Li J, Pfeiffer RM, Wang Y, Guida JL et al. Breast cancer risk factors and mammographic density among high-risk women in urban China. NPJ Breast Cancer [Internet]. 2018 Feb 6 [cited 2019 May 4]; 4(3): 1-12. Available from: https:// www.ncbi.nlm.nih.gov/pubmed/29423438
5. Prolla CMD, Silva PS, Netto CBO, Goldim JR, Ashton-Prolla P. Conocimiento del cáncer de mama y cáncer de mama hereditario en el personal de enfermería de un hospital público. Rev Lat Am Enfermagem [Internet]. 2015 Ene-Feb [citado 4 May 2019]; 23(1): 90-97. Disponible en: http:// dx.doi.org/10.1590/0104-1169.0185.2529

6. Aguirre-Loaiza HH, Núñez C, Navarro AM, Cortés Sánchez S. Calidad de vida según el estadio del cáncer de seno en mujeres: análisis desde el FACT-B y SF-36. Psychol. av. discip. [Internet]. 2017 Ene-Jun [citado 4 May 2019]; 11(1): 109-120. Disponible en: http://www.scielo. org.co/scielo.php?script=sci_abstract $\&$ pid $=\$ 1900$ 23862017000100109\&lng=en\&nrm=iso\&tlng=es

7. Ministerio de Salud. Guías Clínicas AUGE Cáncer de Mama [Internet]. Santiago: Minsal; 2015 [citado 20 Mar 2019]. 164 p. Disponible en: https://www.minsal. cl/wp-content/uploads/2015/09/GPC-CaMama.pdf

8. Caniçali Primo C, Nunes Gonçalves LR, Pires Olympio PC de A, Costa Leite FM, Costa Amorim MH. Ansiedad en mujeres con cáncer de mama. Enferm. Glob. [Internet]. 2012 Oct [citado 18 Jun 2017]; 11(28): 52-62. Disponible en: http://scielo. isciii.es/scielo.php?script=sci_arttext $\&$ pid $=\$ 1695$ $-61412012000400005$

9. Icaza G, Núñez L, Bugueño H. Descripción epidemiológica de la mortalidad por cáncer de mama en mujeres en Chile. Rev Med Chil [Internet]. 2017 [citado 4 May 2019]; 145: 106114. Disponible en: http://dx.doi.org/10.4067/ S0034-98872017000100014

10. Ministerio de Salud, Departamento de Manejo Integral del Cáncer y otros Tumores. Plan Nacional del Cáncer 2018-2028 [Internet]. Santiago: Minsal; 2018 [citado 20 Mar 2019]. p. 38-41 Disponible en: https://www.minsal.cl/ wp-content/uploads/2019/01/2019.01.23_PLANNACIONAL-DE-CANCER_web.pdf

11. Serra I, Maya D, Aguayo C. Cáncer de mama en Chile: mortalidades comparadas: niveles nacional, regional y comunal, 1990-2011. Análisis programático. Rev Chil Cir [Internet]. 2014 Abr [citado 22 Mar 2019]; 66(2): 118-126. Disponible en: https://scielo.conicyt.cl/scielo.php?script=sci_ arttext\&pid=S0718-40262014000200005

12. Ministerio de Salud. Ley Na 19.966. [Internet]. Santiago: Minsal; 2004 [citado 4 May 2019]. 25 p. Disponible en: http://www.supersalud.gob.cl/ normativa/668/w3-article-554.html

13. Feo R, Kitson A, Conroy T. How fundamental aspects of nursing care are defined in the literature: A scoping review. J Clin Nurs [Internet]. 2018 Jun [cited 2019 May 4]; 27(11-12): 2189-2229. Available from: https://www.ncbi.nlm.nih.gov/ pubmed/29514402 
14. Comisión Nacional de Acreditación. Criterios de evaluación para la acreditación de carreras de Enfermería 2007. [Internet]. Santiago: CNA; 2007 [citado 4 May 2019]. 24 p. Disponible en: https:// www.cnachile.cl/Criterios\%20de\%20carreras/ enfermeria.pdf

15. Páez A, Urrea E, Contreras M, Díaz M. Conocimiento y frecuencia de los factores de riesgo para cáncer de mama en las estudiantes del área de la salud. IEID [Internet]. 2011 Mar 24 [citado 4 May 2019]; 12(2): 25-26. Disponible en: https://revistas. javeriana.edu.co/index.php/imagenydesarrollo/ article/view/1153

16. Ecker BL, Lee JY, Sterner CJ, Solomon AC, Pant DK, Shen F, et al. Impact of obesity on breast cancer recurrence and minimal residual disease. Breast Cancer Res [Internet]. 2019 Mar 13 [cited 2019 May 4]; 21(1): 41. Available from: https:// www.ncbi.nlm.nih.gov/pubmed/30867005

17. Martín M, Herrero A, Echavarría I. El cáncer de mama. Arbor [Internet]. 2015 [citado 9 Nov 2017]; 191(773): a234. Disponible en: http://dx.doi. org/ 10.3989/arbor.2015.773n3004

18. Jerônimo AF, Freitas ÂG, Weller M. Risk factors of breast cancer and knowledge about the disease: an integrative revision of Latin American studies.
Cien Saude Colet [Internet]. 2017 Jan [cited 2019 May 4]; 22(1): 135-149. Available from: https:// www.ncbi.nlm.nih.gov/pubmed/28076537

19. Álvarez-Hernández $\mathrm{C}$, Brusint $\mathrm{B}$, Vich P, DíazGarcía N, Cuadrado-Rouco C, HernándezGarcía M. Actualización del cáncer de mama en atención primaria (IV/V). Semergen. [Internet]. 2015 Ene-Feb [citado 8 Nov 2017]; 41(1): 3447. Disponible en: https://doi.org/10.1016/j. semerg.2014.03.004

20. Riquelme B, Sepúlveda B, Vargas X. Atención de enfermería en las pacientes con cáncer de mama. Rev Med. Clin. Condes. [Internet]. 2006 Dic 5 [citado 8 Nov 2017]; 17(4): 244-247. Disponible en: http://www.clc.cl/Dev_CLC/media/Imagenes/ PDF\%20revista\%20m\%C3\%A9dica/2006/4\%20 oct/Atenciondeenferemeriap_244-15.pdf

21. Borré-Ortiz M, Prada-Reyes S, Arrieta-Paternina A, De la Hoz-Pantoja Y, Jiménez-Maury Yur, SantosPuello C. Importance of nursing care in patients with breast cancer: A narrative review. Salud, Barranquilla [Internet]. 2018 Aug [cited 2019 March 20]; 34(2): 475-493. Available from: http://www. scielo.org. co/scielo.php?script $=$ sci_arttext $\&$ pid $=S 0120$ 55522018000200475\&lng=en. http://dx.doi.org/10. 14482/sun.34.2.616.99. 